\title{
Aprovechamiento de las cenizas volantes, clase F, de centrales térmicas para la fabricación de materiales cerámicos
}

\author{
J.M. RUIZ-ROMÁN, C. ALONSO SANTOS, L.E.G. CAMBRONERO, F. CORPAS', M. ALFONSO, A.J. MORAÑO \\ Universidad Politécnica de Madrid. E.T.S.I.Minas, 28003 Madrid \\ ${ }^{1}$ Universidad de Jaén. E.U.P. Linares. 23700 Linares
}

\begin{abstract}
En el presente trabajo se ha investigado la posible utilización de un residuo de las centrales térmicas de carbón de hulla, como son las cenizas volantes silicoaluminosas (clase F) ocasionadas en los procesos de combustión, como materia prima para la fabricación de materiales cerámicos estructurales.De este modo se ha procedido a la caracterización del polvo de partida para la posterior fabricación de un material cerámico mediante compactación y sinterización. Por último, se procedió a la evaluación de diversas propiedades físicas y mecánicas como son la densidad, variación dimensional, absorción de agua y resistencia a flexión de los materiales obtenidos.
\end{abstract}

\section{Palabras clave: ceniza volante, material cerámico, sinterización}

\section{Recovery and reuse of fly ashes (class F) waste for ceramic manufactures}

In the present work we have studied the feasibility of Powder Metallurgy in order to obtain structural ceramics from residual fly ashes powders. Characterization of powders has been carried out followed with the manufacturion of a structural ceramic through poeder compacting and sintering. Some physical and mechanical properties as density, dimensional changes and transverse rupture strength of the obtained materials were evaluated.

Key words: fly ash, ceramic, sintering

\section{INTRODUCCIÓN}

El uso del carbón como combustible para la producción de energía termoeléctrica apareja la generación de grandes cantidades de residuos sólidos conocidos como cenizas volantes, las cuales aunque son mayoritariamente vertidas a escombrera, por sus propiedades tienen una gama de posibilidades para ser usadas a escala industrial, existiendo hoy día aplicaciones ya tradicionales de este material, extendidas por todo el mundo.

En líneas muy generales $(1,2)$, las cenizas volantes son materiales pulverulentos de granulometría muy fina (mayoritariamente en el rango de $1 \mu \mathrm{m}$ a $100 \mu \mathrm{m}$ ), de naturaleza heterogénea, compuestas por partículas vítreas de forma esférica o redondeada, procedentes de la fusión prácticamente completa de los minerales que constituyen la materia mineral (ceniza) del carbón. Su composición química está conformada por cinco componentes mayoritarios: sílice, alúmina, óxidos de hierro y óxidos de calcio y magnesio, junto con una serie de componentes minoritarios (sodio, potasio, titanio, fósforo, carbon inquemado y azufre en forma de sulfatos) y trazas de prácticamente la totalidad de los restantes elementos de la Tabla Periódica. En general, el 50\% de las partículas tienen un diámetro equivalente inferior a $30 \mu \mathrm{m}-40 \mu \mathrm{m}$. La superficie específica oscila de $2500 \mathrm{~cm}^{2} / \mathrm{g}$ a $5000 \mathrm{~cm}^{2} / \mathrm{g}$. La densidad varía de $2,2 \mathrm{~g} / \mathrm{cm}^{3}$ a $2,8 \mathrm{~g} / \mathrm{cm}^{3}(3,4)$.

La ceniza volante proviene de la transformación física, química y mineralógica de la materia mineral del carbón, una vez sometida a las fuertes velocidades de calentamiento y a las altas temperaturas que se alcanzan en el interior del hogar. Se obtienen por precipitación electrostática o mecánica de partículas pulverulentas arrastradas por los flujos gaseosos de calderas alimentadas con carbón pulverizado.

Durante la combustión del carbón, la materia orgánica quema rápidamente, aunque no en su totalidad, permaneciendo como inquemado en la ceniza. La materia inorgánica, por el contrario, sufre transformaciones mas o menos complejas: deshidrataciones, descarbonataciones, desulfuraciones e, incluso, volatilizaciones $(5,6)$.

Los restos de las partículas más groseras funden, se aglomeran y se depositan en el hogar, en tanto que las partículas más finas permanecen en suspensión en la corriente gaseosa como partículas fundidas. Enfriadas rápidamente quedan como esferas vítreas, con parte de fracción cristalizada en las partículas de mayor tamaño.

El importante aumento de la producción mundial de cenizas volantes en los últimos quince años, motivados por los cambios de las estructuras energéticas de aquellas regiones que dependían fuertemente de la importación de productos petrolíferos para la producción eléctrica, y que han llegado actualmente a consumir mayoritariamente carbón, ha condicionado un creciente interés por la magnitud del problema medioambiental asociado a ese residuo.

En la mayoría de las centrales térmicas (7), un mínimo del $70 \%-75 \%$ de la ceniza presente en el carbón, se recoge en forma de cenizas volantes, y por término medio, se producen 
alrededor de $700 \mathrm{t}$ anuales de cenizas volantes por MW de potencia instalada, lo que llega a significar una producción total estimada de cenizas volantes en España de 7,5 x 10 $\mathrm{t} /$ año.

Este trabajo de investigación tiene como objetivo principal dar una salida a un producto residual cuyo volumen es importante y creciente y así facilitar un futuro aprovechamiento, además de reducir su impacto ambiental.

\section{PROCEDIMIENTO EXPERIMENTAL}

Las cenizas volantes empleadas como polvo de partida para la fabricación de los materiales cerámicos (8) presentan una granulometría inferior $200 \mu \mathrm{m}$, teniendo el 50\% de las partículas un diámetro equivalente inferior a $40 \mu \mathrm{m}$. Su superficie específica oscila entre $2500 \mathrm{~cm}^{2} / \mathrm{g}$ a $5000 \mathrm{~cm}^{2} / \mathrm{g}$ y su densidad se encuentra dentro del intervalo $2,2 \mathrm{~g} / \mathrm{cm}^{3}-2,8 \mathrm{~g} / \mathrm{cm}^{3}$. En la tabla I se muestran los resultados obtenidos de los ensayos químicos realizados sobre las cenizas utilizadas en este trabajo con el fin de determinar su composición .

La cera empleada como aglomerante fue la HOESCHT WASH C bajo patente de la misma casa. Algunas características de esta cera son una densidad de $1 \mathrm{~g} / \mathrm{cm}^{3}$ y un tamaño de partícula $(50 \%)<40 \mu \mathrm{m}$. La mezcla se llevo a cabo a temperatura ambiente durante 20 minutos en un mezclador manual en las proporciones $94 \%$ en peso de polvo de ceniza volante y $6 \%$ en peso de polvo de cera. La consolidación de los polvos se realizó en una prensa de 150 toneladas mediante compactación uniaxial en matriz flotante. La mezcla ceniza-cera se compactó a una presión de $200 \mathrm{MPa}$ en una matriz rectangular de $2 \mathrm{~cm} \times 1 \mathrm{~cm}$. La sinterización de las muestras se llevo a cabo al aire sin necesidad de tener atmósfera controlada debido a la naturaleza del material a estudiar. Se sinterizaron probetas durante 30 minutos a las siguientes temperaturas: $1050^{\circ} \mathrm{C}, 1150^{\circ} \mathrm{C}, 1175^{\circ} \mathrm{C}$ y $1200^{\circ} \mathrm{C}$, con velocidades de calentamiento y enfriamiento de $5^{\circ} \mathrm{C} / \mathrm{min}$.

\section{RESULTADOS Y ANÁLISIS}

Las propiedades de los materiales obtenidas en función de su temperatura de sinterización quedan recogidas en las tablas II y III.

El estudio de los resultados obtenidos a partir de los distintos ensayos realizados sobre los distintos materiales, se centra en el análisis de la distribución, número y tamaño de poros, y tamaño de grano del material sinterizado. Para apoyar este razonamiento se realizó un estudio mediante microscopía electrónica de barrido sobre muestras sinterizadas a las diferentes temperaturas indicadas con anterioridad. Se seleccionaron dos aumentos diferentes con el objetivo de poder comprobar fundamentalmente el grado de sinterización alcanzado, el tamaño y número de poros y el tamaño de grano de las muestras.

El interés de controlar el crecimiento de grano en los procesos de fabricación de los materiales cerámicos se deriva de dos causas fundamentales. Una primera es que va a ser uno de los factores fundamentales que va por si mismo a determinar las propiedades finales del componente, y la otra es que de su control va a depender poder alcanzar componentes de densidades próximas a la teórica lo cual redunda en una mejora de las propiedades de los mismos.

El tamaño de grano puede afectar a las propiedades del componente final bien porque condiciona la medida sobre otros parámetros del material (por ejemplo el desajuste entre
TABLA I. COMPOSICIÓN QUÍMICA DEL POLVO DE CENIZA VOLANTE EMPLEADO COMO MATERIA PRIMA PARA LA FABRICACIÓN DE LOS MATERIALES CERÁMICOS OBJETO DE ESTE TRABAJO.

\begin{tabular}{|l|c|c|}
\hline ENSAYO & NORMA & F1 (\%) \\
\hline $\mathrm{SiO}_{2}$ reactiva & UNE 80-225 & 37,40 \\
\hline Alúmina & UNE-EN 196-2 & 22,57 \\
\hline $\mathrm{SiO}_{2}+\mathrm{Al}_{2} \mathrm{O}_{3}$ & & 59,97 \\
\hline Perdida por calcinación bruta & EN 196-2 & 2,25 \\
\hline
\end{tabular}

TABLA II. PROPIEDADES FÍSICAS Y MECÁNICAS DE LOS MATERIALES RESULTANTES EN FUNCIÓN DE SU TEMPERATURA DE SINTERIZACIÓN.

\begin{tabular}{|c|c|c|c|}
\hline $\mathrm{T}^{\mathrm{a}}$ sinterización & $\begin{array}{c}\text { Densidad } \\
\left(\mathrm{g} / \mathbf{c m}^{\mathbf{3}} \mathbf{)}\right.\end{array}$ & $\begin{array}{c}\text { Variación } \\
\text { dimensional (\%) }\end{array}$ & $\begin{array}{c}\text { Resistencia a } \\
\text { flexión } \mathbf{( M P a )}\end{array}$ \\
\hline $1050^{\circ} \mathrm{C}$ & 1,742 & -10 & 20,0 \\
\hline $1150^{\circ} \mathrm{C}$ & 1,759 & -18 & 34,4 \\
\hline $1175^{\circ} \mathrm{C}$ & 1,785 & $-19,1$ & 45,6 \\
\hline $1200^{\circ} \mathrm{C}$ & 1,804 & $-20,3$ & 45,2 \\
\hline
\end{tabular}

TABLA III. ReSUlTADOS DE LOS ENSAYOS DE ABSORCIÓN DE AGUA

\begin{tabular}{|l|c|c|c|c|}
\hline $\begin{array}{c}\mathrm{T}^{\mathrm{a}} \text { sinterización } \\
\text { seco (g) }\end{array}$ & $\begin{array}{c}\text { Peso en } \\
\text { absorción (g) }\end{array}$ & $\begin{array}{c}\text { Peso tras } \\
\text { de agua (g) }\end{array}$ & $\begin{array}{c}\text { Absorción } \\
\text { de agua (\%) }\end{array}$ & Absorción \\
\hline $1050^{\circ} \mathrm{C} 2,904$ & 3,297 & 0,393 & 13,55 & \\
\hline $1150^{\circ} \mathrm{C} \quad 2,779$ & 2,917 & 0,138 & 4,97 & \\
\hline $1175^{\circ} \mathrm{C} \quad 2,901$ & 2,998 & 0,097 & 3,34 & \\
\hline $1200^{\circ} \mathrm{C} \quad 2,933$ & 2,994 & 0,061 & 2,07 & \\
\hline
\end{tabular}

granos que crecen a partir de una expansión térmica anisotrópica), bien porque fija la cantidad de bordes de grano y sus diferentes propiedades. Del análisis microestructural se puede comprobar que el tamaño de grano inferior corresponde a los materiales sinterizados a la temperatura más baja $\left(1050^{\circ} \mathrm{C}\right)$, lo que debería repercutir en unas mejores propiedades. Pero esto no es así ya que también se observa en estos materiales que la sinterización ha sido algo incompleta, presentando una porosidad muy elevada que empeora notablemente las propiedades de los mismos. Esta porosidad, además, es de tipo interconectada y por ello se alcanza una elevada absorción de agua en los materiales sinterizados a $1050{ }^{\circ} \mathrm{C}$.

En cuanto a la porosidad de los otros materiales obtenidos, como era de esperar disminuye con el aumento de la temperatura de sinterización posiblemente por la existencia de una fase líquida, pudiendo observarse una cantidad importante de poros aislados intragranulares en los materiales sinterizados a $1200{ }^{\circ} \mathrm{C}$. Si a este fenómeno le añadimos los resultados obtenidos en las otras propiedades evaluadas (densidad, absorción de agua, variación dimensional y resistencia a flexión), podemos concluir que la temperatura que se muestra como óptima para sinterizar estos materiales se situaría próxima a los $1175^{\circ} \mathrm{C}$.

La segunda razón para el control del crecimiento de grano se encuentra en la búsqueda de una elevada densidad durante la sinterización. El reconocimiento de que la densificación sólo puede derivarse de una razonable relación de fuentes y sumideros para los procesos de difusión asociados, y más particularmente, la identificación de los bordes de grano y poros como esas fuentes y sumideros de átomos de difusión, han dado lugar a la creencia de que la total densificación sólo puede esperarse donde los poros permanezcan retenidos por los bordes de grano. Dicho esto, queda claro 
que un crecimiento anormal de los granos donde los poros quedan aislados debe evitarse si lo que se busca es obtener cerámicas de elevada densidad, lo que hace que en principio se descarte la temperatura de $1200{ }^{\circ} \mathrm{C}$ como la óptima de sinterización.

El objetivo que se persigue a la hora de obtener elevadas densidades es mejorar los resultados en propiedades afectadas por la porosidad. Por ejemplo, los poros actúan como concentradores de tensiones con el consiguiente efecto contrario en las propiedades mecánicas, provocan un efecto adverso sobre la permeabilidad y también perjudica a las propiedades ópticas en particular a la transmisión de la luz. No obstante, en el caso de buscarse bajas densidades y aislamiento térmico, la porosidad puede ser beneficiosa.

En el caso de la resistencia a fractura, su valor en función de la porosidad puede expresarse por la siguiente relación:

$$
\sigma=\sigma_{\mathrm{o}} \cdot \mathrm{e}^{-n v}
$$

donde:

n es una constante que toma el valor entre 5 y 7

$\mathrm{v}$ es la fracción volumétrica de la porosidad

Una porosidad del $10 \%$ reduce a la mitad la resistencia a rotura. En el caso del módulo de elasticidad, este también se ve afectado por la porosidad según la relación:

$$
\mathrm{E}=\mathrm{E}_{\mathrm{o}} \cdot\left(1-1,9 \mathrm{~V}+0,9 \mathrm{~V}^{2}\right)
$$

También la forma de los poros será importante ya que la concentración de tensiones tomará valores altos para elipses con el eje mayor normal a la dirección del campo de tensiones, y bajos para poros esféricos pequeños.

Podemos por tanto justificar los resultados obtenidos en los diferentes materiales por dos hechos fundamentales que quedan demostrados en el análisis microestructural:

Una disminución de la porosidad conforme aumentamos la temperatura de sinterización, mostrándose como temperatura más adecuada de sinterización $1175^{\circ} \mathrm{C}$.

Una esferoidización de los poros conforme la temperatura de sinterización aumenta, aunque en el caso de la temperatura de $1200^{\circ} \mathrm{C}$ se produce un importante número de porosidad aislada dentro de los granos.

Las propiedades evaluadas para los distintos materiales cerámicos resultantes en función de su temperatura de sinterización se recogen en la figura 1, en la que se observa como se alcanza el máximo de propiedades para una temperatura de sinterización de $1175^{\circ} \mathrm{C}$.

Como conclusión, la tecnología de partículas se ha mostrado como un proceso eficaz para la obtención de materiales cerámicos estructurales a partir de polvos residuales de cenizas volantes de centrales térmicas, lo que puede constituir una alternativa económica al vertido de dichos residuos.

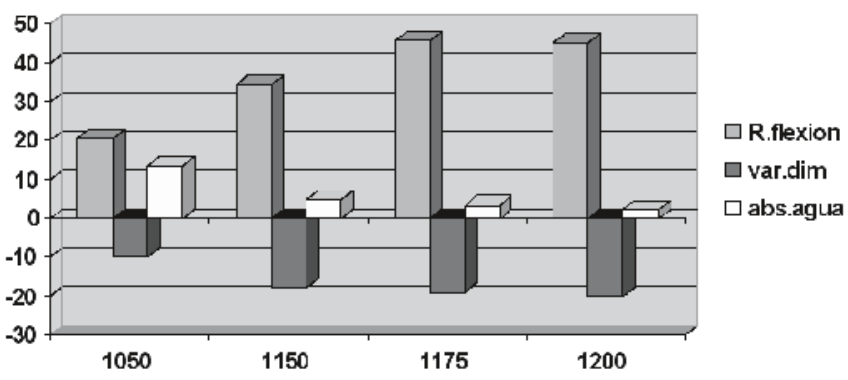

Figura 1. Resumen de resultados de los materiales obtenidos en función de su temperatura de sinterización.

\section{CONCLUSIONES}

La Tecnología de Partículas se ha mostrado como un proceso eficaz para la obtención de materiales cerámicos estructurales a partir de polvos residuales de cenizas volantes de centrales térmicas, lo que puede constituir una alternativa económica al vertido de dichos residuos.

Para el conformado en matriz uniaxial de los polvos de ceniza volante es necesario adicionar un aglomerante en cantidades en torno al 5\% dependiendo de la pieza a obtener.

La temperatura de sinterización óptima para los materiales obtenidos es de $1175{ }^{\circ} \mathrm{C}$ durante 30 minutos en atmósfera de aire.

Los resultados obtenidos abren la posibilidad de que este proceso pueda trasladarse a la industria de modo que se puedan fabricar tejas, losetas o ladrillos a partir de estos residuos industriales.

\section{BIBLIOGRAFÍA}

1. Cañadas-Serrano, L y otros. “Caracterización de las cenizas de cinco centrales térmicas españolas", Medio ambiente- RETEMA, 13-20 (1990).

2. De Luxan, M.P., Sánchez Rojas, I. y Soria, F. “Características de Cenizas volantes Españolas", Mater. de const. Vol. 38, 209, 25-38 (1988).

3. Acha, M. "Aplicaciones de las cenizas volantes diferentes a las utilizadas en la industria de la construcción". Energía, 26, Enero-Febrero, 47-51 (1996).

4. Moya, J.S. y Morales, F. “Utilización de los barros rojos de la plantas de alúmina", Bol. Soc. Esp. Cerám. y Vidrio, 26, 1, 21-30 (1987).

5. Gómez Cañibaro, J. “Pruebas de laboratorio con los estériles de menudos del carbón para la fabricación de materiales cerámicos". Bol. Soc. Esp. Cerám. y Vidrio, 25, 4, 243 (1987).

6. Geiger, G. "Powder Synthesis and Shape Forming of Advanced Ceramics". Am. Ceram. Soc. Bull., 74, 8, 77-80 (1995).

7. Del Amor García, G. “Estudio sobre la adición de cenizas volantes al hormigón". Proyecto Fin de Carrera. E.T.S. Ingenieros de Minas de Madrid (1997).

8. Lee, W.E. \& Mark Rainforth, W. Ceramic Microstructures. Property Control by Processing. Ed. Chapman \& Hall (1994). 